\title{
The Sagnac effect proves that Einstein's "constant speed of light" is wrong Duan Xian Xiang
}

\begin{abstract}
The theory of relativity is based on the principle of "constant speed of light", but the Sagnac effect proves that the constant speed of light is wrong, and the "ether" experiment of Michelson and morley should not be zero.
\end{abstract}

Key words relativity theory, constant velocity of light, Sagnac effect, Michelson experiment

The introduction

One hundred years ago, Einstein proposed the special theory of relativity and the general theory of relativity. The most important basis of these two theories is the principle that the speed of light does not change. The explanations for this experiment are "the speed of light remains constant" and "the ether does not exist".

According to the paper, the Michelson and morley experiments produced zero results because the interferometer was stationary on the ground and did not move.

\section{Ether model}

This paper presents an "ether" model.

Every planet and even object has its "ether", which is relatively stationary with this planet and follows its rotation and revolution. This "etheric" wraps the planet, the closer you get to a star, the denser the ether becomes; This "ether" is most likely the planet's gravitational field.

This model can be used to explain the zero results of Michelson experiment, the Sagnac effect, and the "starlight deflection" and "gravitational lens".

2. The interpretation of the zero result of Michelson and morley experiment

Because the etheric around the earth follows the earth's rotation and revolution, the etheric and the earth are relatively stationary (There is also etheric around the sun, which is relatively static with the sun), and Michelson's interferometer is fixed on the ground, its interference fringes do not change when rotating the interferometer, because the etheric and the interferometer are relatively stationary.

This creates the "constant speed of light" illusion.

3. The Sagnac effect

Sagnac Effect is the invention of a rotating annular interferometer by Sagnac in 1913. Splitting a beam of light from the same light source into two beams, making them converge in the same loop in opposite directions for one week, and then producing interference on the screen. When there is rotational angular velocity in the loop plane, interference fringes on the screen will move, which is known as the sagnak effect.

Sagenak effect has been widely used. Fiber optic gyroscope developed by sagenak effect ct has been successfully used in aviation, aerospace and other fields.

Here, the Sagnac effect appears in the rotating system, but this paper holds that the Sagnac effect has nothing to do with the "rotating system" or the "inertial system". The Michelson-Morey experimental interferometer is placed on the aircraft to make the interferometer in the inertial system, and the Sagnac effect will also appear in the interferometer.

The Sagnac effect is the same as that of Michelson's-morley's experimental interferometers, except that the gyroscope is in motion and the Michelson 
interferometer is stationary. The motion of the interferometer has streaks, indicating that the ether is stationary relative to the earth.

This effect indicates that the zero result of the Mm experiment is because the interferometer does not move. If the $\mathrm{Mm}$ interferometer moves relative to the ground, the interference fringe will move, so there will be no zero result, and thus no "constant speed of light".

For this problem, we can do an experiment, namely, the michelson-morley experiment of "the motion of the interferometer relative to the ground". If the interferometer moves and turns the interferometer 90 degrees, the interference fringes will change.

In fact, the Sagnac effect is one such experiment.

Conclusion: since the Sagnac effect proves that the speed of light does not change is wrong, Einstein's theory of relativity, which is based on the assumption that the speed of light does not change, is also wrong. 